\title{
3 "A materials way of thinking": Chiralität in Nano-, Material- und Oberflächenforschung
}

Als erste Fallstudie zu den Re-Konfigurationen der chemischen Felder werden in diesem Kapitel die Materialwissenschaften und ihre gegenwärtigen Beiträge zur Chiralitätsforschung diskutiert. In diesem multidisziplinären und hybridisierten Forschungsbereich zeigt sich wohl am deutlichsten, wie die Aneignung und Umdeutung tradierter, chemischer Gegenstände wie der molekularen Chiralität durch die physikalischen Wissenschaften vonstatten geht. Mit der Erschließung besonders kleiner und spezifischer Sonderformen der Materie, beispielsweise Nanopartikeln und -strukturen sowie der supramolekularen Ebene, werden sukzessive physikalische Weltbilder auf etablierte, chemische Gegenstände und Fragestellungen dominant. Dies zeigt sich etwa darin, dass der Begriff der Chiralität auf „im strengen Sinne “1 nicht-chirale Gegenstände wie Elektrospin und zweidimensionale Oberflächenstrukturen angewandt wird und zudem mit physikalischen Konzeptionen wie der Helizität amalgamiert. Die Materialwissenschaften, die über mehrere Jahrhunderte hinweg primär ein chemisches Betätigungsfeld darstellten, werden seit den 1960er Jahren zunehmend von Physiker/innen adaptiert, was sich heute in einem ambivalenten Verhältnis zwischen den Fachkulturen ausdrückt und Gegenstand der Grenzarbeit der chemischen Felder ist. Einerseits verspricht die Nähe zur Physik einen Zugewinn an Übereinstimmung mit impliziten Präideen der Einheit und Hierarchie der Wissenschaften, gemäß deren Vorstellungen ein physikalischer Zugriff auf die Natur als überlegen gilt. Auf der anderen Seite reagieren Chemiker/innen in diesem Feld allerdings auch im Sinne einer "conquest of [their] own territory“ (Bensaude-Vincent und Stengers, 1996, S. 208f.), wenn chemische Ansätze unsichtbar und chemische Gegenstände von außen angeeignet werden:

Diese Formulierung geht auf das Interview mit dem Materialwissenschaftler Prof. Borodin zurück, der mit der entsprechenden Semantik der Unterscheidung echter und falscher, bzw. chemischer und nicht-chemischer Chiralität ein Leitmotiv für die Grenzarbeit zwischen Chemie und Physik im hybriden Feld der Nano- und Materialwissenschaften liefert. 
Chemieingenieur Prof. Borodin: Ein berühmterChemiker, der die Katalyse mitbegründet hat, Wilhelm Ostwald, hat immer gesagt: „ein Chemiker der nicht auch Physiker ist, ist GAR nix". Das heißt, Chemiker müssen Physik LERNEN. Die machen's nicht so gut wie die Physiker aber sie KÖNNEN'S. Also das heißt, sie können ein bisschen Physik und CHEMIE. Ein PHYSIKER ist völlig ignorant der Chemie gegenüber. Also das [ist] verallgemeinernd jetzt aber das heißt, die haben CAR keine Ahnung von Chemie und sie lernen's auch nicht und sie halten's auch nicht mal für nötig. Aber sie GEHEN dann in das Cebiet der Chemie, sie benutzen Moleküle, Materialien und haben keine Ahnung und fangen dann an da rumzustochern. Als Chemiker hat man dann bestimme Sachen schon gelernt, erkannt, definiert, BENANNT und die erfinden das Rad neu weil sie zu arrogant sind, mal in andere Disziplinen zu schauen. Also ich find des erschreckend.

$\mathrm{Zu}$ Beginn der 2000er Jahre galten unter Historiker/innen und Philosoph/innen der Chemie die Material- und Nanowissenschaften als paradigmatische Bereiche, in denen sich die Re-Konfigurationen der chemischen Felder - und vor allem deren Auflösung in hybride Disziplinen - beobachten ließen (Bensaude-Vincent, 2008). Es entspann sich eine Kontroverse darum, ob es sich bei den Feldern um eigenständige „Superdisziplinen“ mit einer geteilten Fachidentität, theoretischen Konzepten, Methoden und Fragestellungen handle (Hentschel, 2011) oder um einen losen, desintegrierten Flickenteppich vorwiegend regional organisierter Wissensproduktion (Bensaude-Vincent, 2001). Auch die Science and Technology Studies zogen die Nano- und Materialforschung für zahlreiche Fallstudien zum Studium disziplinärer Dynamiken heran. Sie spielten in dieser Forschungstradition eine bedeutende Rolle in der Theoriebildung zur disziplinären Identität (Kurath und Kaiser, 2010), fachspezifischen Denkstilen (Schaper-Rinkel, 2007), Inter- und Transdisziplinarität (Schummer, 2004a) sowie zu den sozialen Bedingungen der Wissensproduktion vor dem Hintergrund zeitgenössischer Innovationsdispositive. $^{2}$

Der zentrale Befund dieses Kapitels trägt zur Debatte um die Grenzziehung wissenschaftlicher Disziplinen angesichts ihrer Hybridisierung im Zuge sich wandelnder Innovationsdispositive bei: Die Erforschung, Entwicklung, Produktion,

Im Rahmen der Programmatik sog. konvergierender Technologien wurden seit der Jahrtausendwende anwendungsorientierte, gegenstandsbezogene und interdisziplinäre Disziplinen der Nano-, Bio- und Informationstechnologie neben den Neurowissenschaften (NBIC) im Rahmen nationaler Innovationsstrategien mit enormen Summen gefördert (BensaudeVincent, 2016, S. 51). Die entsprechenden politischen Initiativen der USA und der EU entsprechen einer Wissenschaftspolitik der "commercialized postmodern university" (Mody und Choi, 2013, S. 125), die sich vor allem durch einen unternehmerischen Innovationsgedanken auszeichnet (Kooperation mit Privatwirtschaft, erhöhte Konkurrenz und Wettbewerb, Forderung nach gesellschaftlich und wirtschaftlich relevanter Forschung, Großprojekte) (ebd., S. 130ff.). 
Prüfung, Optimierung, Wiederverwertung und Vernichtung von Materialien war über mehrere Jahrhunderte durch einen primär chemischen Zugriff auf die mikroskopischen Dimensionen von Materie und Materialien geprägt (Hentschel und Reinhardt, 2011, S. 2). Diese Dominanz ist allerdings seit dem Aufkommen der Nanowissenschaften und surface sciences im Schwinden begriffen, zumal physikalische und informationstechnologische Zugriffe auf das Material an epistemischer Deutungsmacht zunehmen (Küchler, 2015, S. 273). Obgleich diese Modi des Erkenntnisgewinns im Kontext der entstehenden Bergakademien des ausgehenden 18. und der Materialprüfanstalten des 19. Jahrhunderts interdisziplinär angelegt waren, rekrutierte sich die leitende Expertise überwiegend aus den chemischen Feldern (Klein, 2016, S. 188). Die zentrale Heuristik dieses chemischen Zugriffs auf die Struktur von Metallen, Keramiken, Mineralen und später auch Kunststoffen unterschied sich allerdings deutlich von den aus Kapitel 2 bekannten molekularen Modellen der organischen Synthesechemie (Glazer, 2016, S. 7f.). Die atomare Gitterstruktur des Kristalls bzw. des Festkörpers unter Berücksichtigung entsprechender physikalischer Eigenschaften wie elektrische Leitfähigkeit, Formbarkeit oder Magnetismus stellten das erkenntnisleitende Modell der überwiegend anorganischen Festkörperchemie dar, die die Wissensproduktion in der Materialforschung anführte (Urban, 2015, S. 8, 50).

Dass die etablierte Grenzlinie zwischen chemischer Struktur und physikalischen Eigenschaften und Feldern allmählich erodiert, zeigt sich am Fallbeispiel der Chiralität. Die Spiegelasymmetrie zeigt sich in den Materialwissenschaften auf der Ebene von asymmetrischen kristallinen Gebilden und Kristallisationsprozessen sowie Absorptionsprozessen von Molekülen an Oberflächen. Während Chemiker/innen dazu neigen, die Definition der Chiralität auf diese Themenbereiche (neben den Strukturmerkmalen organischer Moleküle) zu beschränken, zeigt sich, dass Physiker/innen den Begriff der Chiralität auf verschiedene Phänomenbereiche ausweiten, die der etablierten chemischen Konzeption nicht entsprechen. Zunächst folgt in Abschnitt 3.1 eine historische Hinführung zum Thema, in der erörtert wird, wie sich physikalische und chemische Zugriffe auf Materialien und Werkstoffe unterscheiden und wie sich ihr Verhältnis im Zuge der wechselvollen Geschichte der Materialwissenschaften als interdisziplinäres Forschungsfeld darstellt. In den Abschnitten 3.2 und 3.3 werden in diesem Zusammenhang erstens das physikalische Konzept zweidimensionaler Chiralität und zweitens die Chiralitätskonzepte in Bezug auf magnetische Oberflächen angeführt: In beiden Fällen handelt es sich um physikalische Aneignungen chemischer Prozesse, denen Chemiker/innen in ihren Abgrenzungsstrategien entschiedenen widersprechen. 


\subsection{Physik oder Chemie? Eine kleine Geschichte des Materials}

Im Alltagsverständis von technoscientifischen Innovationen finden Materialien eher wenig Beachtung. In ihrer Unscheinbarkeit fallen sie in der Wahrnehmung hinter denjenigen Produkten zurück, die sich aus ihnen zusammensetzen, allen voran Batterien, Textilien, Motoren, künstliche Gelenke, Speicherchips oder Kunststoffverpackungen (Urban, 2015, S. 1). Auch die Materialwissenschaften als Feld der Erforschung, Entwicklung, Optimierung und Entsorgung von Werkstoffen erfahren erst zögerlich Aufmerksamkeit vonseiten einer interessierten Öffentlichkeit, zumal Materialien im weitesten Sinne heute $\mathrm{zu}$ den größten globalen Märkten zählen, die zudem ein enormes Marktwachstum versprechen und enormen Zuspruch vonseiten der Forschungspolitik und Investor/innen genießen (Küchler, 2015, S. 271). Auch wenn nicht jedes materialwissenschaftliche Entwicklungsprojekt $\mathrm{zu}$ einer Innovation führt, die sich mittel- bis langfristig am Markt etablieren wird, bringt sie dennoch am Markt verwertbare Nebeneffekte hervor: Ständig in der Optimierung begriffene Technologien im Bereich der Herstellung, Analyse und Verarbeitung von Materialien werden in steter Auseinandersetzung mit den Materialien weiterentwickelt und dienen selbst als Quelle der Innovation (ebd.). Auch die Fähigkeit, bestehende Materialien umzuwandeln, wiederzuverwerten, zu entsorgen, $\mathrm{zu}$ vernichten oder für diese erweiterte Anwendungsbereiche $\mathrm{zu}$ erschließen, erhöht das Innovationspotential des Feldes (ebd.). Spezialmaterialien in der industriellen Anwendung verfügen zudem über immer längere und komplexere Wertschöpfungsketten, sie werden smarter, nachhaltiger, liefern Daten für komplexe Algorithmen und entsprechen den zeitgenössischen Innovationsdispositiven des neoliberalen Industriekapitalismus in höchstem Maße (Segal, 2017, S. 10).

Darüber hinaus hat sich in den Materialwissenschaften seit den 1980er Jahren ein neuer Wissenschaftsstil herausgebildet, aus dem sich u.a. auch die Nanowissenschaften speisen. Den Denkstil des „materials way of thinking“, der in einem charakteristischen Triple Helix-Innovationsmodell an der Schnittstelle von Akademie, Privatwirtschaft und Politik verankert ist, impliziert, dass der Innovationsprozess in diesem Feld nicht linear abläuft, sondern kreislauf- bzw. netzwerkförmig (Bensaude-Vincent, 2011, S. 107). Dies bedeutet, dass nicht nur das Zielprodukt Teil des Entwicklungsprozesses ist, sondern auch das Werkzeug selbst, mit dem es hergestellt wird. Der Innovationsprozess ist zudem interdisziplinär, unternehmerisch und auf drängende gesellschaftliche Herausforderungen ausgerichtet (ebd.). Es herrscht allerdings kein Konsens darüber, ob dieser Forschungsstil tatsächliche ontologisch einzigartige, neue Gegenstände hervorgebracht hat, oder ob es sich bei den Nanowissenschaften nicht um alten Wein in neuen Schläuchen handelt (Schummer, 2004a). Auch die tatsächliche Interdisziplinarität wurde angezweifelt 
und ob dieses Feld tatsächlich die versprochenen Innovationen geniert (Marcovich und Shinn, 2011).

Bei den Materialwissenschaften handelt es sich um ein interdisziplinäres Feld, das sich an den Schnittstellen von Physik, Chemie, Elektrotechnik, Maschinenbau, Kristallografie, Geologie und Metallurgie angesiedelt hat (ebd.). Die Materialwissenschaften unterscheiden sich dabei in ihrer disziplinären Ausrichtung und thematischen Schwerpunktsetzung bezüglich lokaler Fachkulturen und Spezifika (Bensaude-Vincent, 2016, S. 51): Je nachdem an welchen Hochschulen, Fakultäten, Forschungsverbünden oder Lehrstühlen die Materialwissenschaften infrastrukturell angegliedert sind, haben sie tendenziell eher einen eher physikalischen bzw. chemischen Zugriff auf die Erforschung und Entwicklung von Materialien (ebd.). Dies rührt daher, dass zwischen dem chemischen Aufbau von Materialien, deren physikalischen Eigenschaften und ingenieurswissenschaftlichen Anwendungsbezügen unterschiedliche Schwerpunkte gesetzt werden (Bensaude-Vincent, 2007, S. 222). Was das Feld eint, ist die hohe industrienahe Anwendungsorientierung (Urban, 2015, S. 3). Innovationen der Materialwissenschaften sowie die Schwierigkeiten ihrer Skalierbarkeit von Prototypen, die unter artifiziellen Laborbedingungen entwickelt werden und auf Industriemaßstab extrapoliert werden sollen, dienen der Technikforschung als paradigmatisches Feld zur Erforschung von Innovationsprozessen (Mody, 2001, S. 10). Bei den Materialwissenschaften handelt es sich um eine vielschichtige Technoscience, bei der der empirische Forschungsgegenstand zugleich durch Verfahren ingenieurstechnischen Entwickelns hervorgebracht und optimiert wird (Mody und Lynch, 2010, S. 16).

,Materials science is an umbrella term for an extraordinary diverse set of practices and occupational and disciplinary affiliations. It includes, but is certainly not limited to, the study of metals, polymers, ceramics, glasses and composites, with respect to their electrical, mechanical, thermal, optical, and other properties [...]. Many who receive the appelation , materials scientist' might alternatively refer to themselves as ,scientists', ,engineers', or as practitioners of more academically traditional disciplines (chemists, physicists, applied physicists, geologists). Alternatively, they may present themselves in terms of the material or application with which they work (ceramists, metallurgists, ,device people', , plastics people' (Mody, 2001, S. 9; 10).

Die Materialwissenschaften haben sich in ihrer Geschichte überwiegend aus den Mutterdisziplinen Chemie und Physik heraus entwickelt bzw. haben sich im Grenzbereich zwischen den überwiegend experimentell orientierten Bereichen der Festkörperphysik und anorganischen Chemie angesiedelt (Schummer, 2004a, S. 15). Es bestehen, wie bereits erwähnt, zahlreiche weitere Überschneidungen mit verschiedenen Ingenieurswissenschaften und in jüngerer Zeit auch den Lebenswissenschaften (Segal, 2017, S. 43ff.). Dabei wurde jedoch von Wissenschafts- 
historiker/innen festgestellt, dass sich eine US-amerikanische und eine europäische Entwicklungslinie des Faches voneinander unterscheiden lassen (BensaudeVincent, 2016). Erstens fand die Entwicklung der beiden Forschungstrends stark zeitversetzt statt, zweitens fiel sie in jeweils unterschiedliche forschungspolitische Kontexte und drittens bildeten jeweils verschiedene Anwendungsfelder, Methoden und Prämissen der Wissensproduktion den Hintergrund. In der Folge seien die beiden Entwicklungslinien der Materialforschung vorgestellt.

Auf dem europäischen Kontinent verfügen die Materialwissenschaften insgesamt über eine längere Tradition, die bis in die Zeit der Frühindustrialisierung zurückreicht. Im ausgehenden 18. Jahrhundert unterliefen zahlreiche Gewerbeund Handwerkszweige einem grundlegenden Wandel: Nicht nur wurde der Maßstab ihrer Produktion angehoben, sondern diese auch in ihrer Herstellung rationalisiert und vereinheitlicht (Urban, 2015, S. 40f.). Die Gewinnung und Verarbeitung von Rohstoffen aus Minen wie Erze, Minerale und fossile Energieträger unterlief einer Verwissenschaftlichung ${ }^{3}$ im Zuge der Entstehung und akademischen Etablierung der chemischen, physikalischen und ingenieurswissenschaftlichen Disziplinen (Vogel, 2013). Von der Begründung sog. Bergakademien als gesonderte Hochschulform erhofften sich die absolutistischen Monarchien Russlands, Österreich-Ungarns, Frankreichs, Preußens und Sachsens nicht nur rationale Kenntnisse über die Zusammensetzung verschiedener Bodenformationen, sondern auch über die Entwicklung von Technologien in der Gewinnung von Bodenschätzen und deren Verarbeitung (Schleiff und Konečný, 2013). Im militärischen, kolonialen wie wirtschaftlichen Wettstreit zwischen den Feudalmächten bildete sich ein Narrativ der Nützlichkeit in Bezug auf die entstehenden Technikwissenschaften heraus, mithilfe dessen sich die Bergakademien von den weniger praktisch orientierten Universitäten und anderen Hochschulformen abgrenzen konnten (Klein, 2016, S. 188). Als weitere Institutionen der Materialforschung etablierten sich im Laufe des 19. Jahrhunderts an verschiedenen europäischen Standorten Materialprüfanstalten, die insbesondere um die Festlegung einheitlicher Qualitätskriterien von Werkstoffen und deren staatlicher Kontrolle bemüht waren (Hentschel, 2011, S. 11f.).

Insbesondere die Produktion von gehärtetem Stahl für den Bau von Eisenbahnen, Brücken sowie dessen Verwendung zu militärischen Zwecken wirkten sich als Treiber der Verwissenschaftlichung der Materialforschung aus. Stahl wurde gewissermaßen zum Leitmaterial der Festkörperchemie (Urban, 2015, S. 41), während die fossile Kohle zum Leitmaterial der organischen Strukturchemie (vgl. Abschnitt 2.2) avancierte: „Die Initialzündung für die wissenschaftliche Untersuchung von Werkstoffen, der Beginn der Werkstoffforschung, wurde jedoch erst durch die industrielle Massenproduktion von Stahl ausgelöst. Handwerker, die bis dahin die Eisen- und Stahlherstellung dominierten und mit ihren Erfahrungen als unersetzbar galten, wurden zunehmend verdrängt von studierten Chemikern, wissenschaftlich orientierten Metallurgen und professionellen Ingenieuren." (Ebd., S. 41). 
Von den Bergakademien ausgehend entwickelten sich im 19. Jahrhundert verschiedene Forschungszweige zur mechanischen und chemischen Gewinnung von Metallen (Metallurgie), der Verhüttung von Eisen-, Silber- oder Uranerzen, sowie deren industriellen Weiterverarbeitung und Qualitätsprüfung (Klein, 2016, S. 70ff.). In Auseinandersetzung mit den Abkühlungsprozessen geschmolzener Metalle entwickelte sich zudem die Kristallografie als Wissenschaft heraus (Glazer, 2016, S. 10ff.). Die Erforschung kristalliner Strukturen und Entwicklung von kristallografischen Technologien fand an mineralischen Stoffen (wie etwa Quarzkristallen) in enger Auseinandersetzung mit der Kristallgitterbildung von verhütteten Metallen statt (Urban, 2015, S. 21). Die Struktur von metallischen und mineralischen Kristallen wurde zudem mit entsprechenden physikalischen Eigenschaften verknüpft, etwa der elektrischen Leitfähigkeit, thermodynamischen Eigenschaften oder auch der optischen Aktivität. Wie in Abschnitt 2.1 deutlich wird, begründet sich auch die Erschließung der chiralen Eigenschaft von Festkörpern in diesen Forschungstraditionen, die ein Bindeglied zu den im Entstehen begriffenen organisch-chemischen Wissenschaften darstellte. Aufgrund dieser Eigenschaften ließen sich kristalline Verbindungen kristallografisch klassifizeren: Raum- und Symmetriegruppen bildeten dabei die primären Klassifikationssysteme der Kristallografie (Glazer, 2016, S. 9). Symmetrieverhältnisse im Allgemeinen und die Chiralität im Speziellen stellen im strukturellen Aufbau des Kristalls zentrale Klassifikationsmerkmale dar (Flack, 2003). Dies wird daher nicht nur in der Geschichte dieser Wissenschaftsbereiche deutlich, sondern auch in der Beobachtung, dass chirale Phänomene ein wachsendes Forschungsfeld in der gegenwärtigen Landschaft der Materialforschung und den Nano Sciences darstellen (Barron, 2009, S. 24).

Die europäische Tradition der Materialwissenschaften sedimentierte sich in der Folge als Disziplinengeflecht mit Wurzeln in verschiedenen Feldern. Die Werkstoffkunde bildet dabei bis heute eine interdisziplinäre Hochschullandschaft mit spezifischen lokalen Fachkulturen, Fachbezeichnungen, Studienprofilen, Industriebeziehungen, Forschungsschwerpunkten und disziplinären Hierarchien (Urban, 2015, S. 2). Insbesondere bezüglich der disziplinären Hierarchien unterscheidet sich die europäische von der US-amerikanischen Tradition: Während sich an den europäischen Hochschulen im Laufe der Zeit ein eher chemisches Verständnis des Festkörpers durchgesetzt hat, dominiert in den USA ein physikalisches (Bensaude-Vincent, 2016, S. 48). Indem physikalische Eigenschaften von Mineralen und Metallen der chemischen Struktur zugeschrieben wurden und physikalische Eigenschaften von Stoffen dem chemischem Aufbau implizit nachgeordnet werden, diente in der europäischen Fachentwicklung tendenziell die Physik als Hilfswissenschaft der chemisch-analytischen Expertise, während in den USA Institute, die den materials sciences \& engineering (MSE) verschrieben waren, deutlich von Experimental- und Festkörperphysiker/innen und deren 
spezifischen Zugriffen auf de Mikrostruktur von Materialien dominiert waren (ebd.). In diesem Fall diente tendenziell die Chemie als Hilfswissenschaft:

\begin{abstract}
Although research on materials developed in many different institutions (including universities, polytechnics and private companies) long before the 1960s, materials science only emerged as an academic entity in the USA, in the context of the Cold War. The scientists who promoted MSE described this research field as the "natural" outcome of physics in the first half of the twentieth century. Their story goes roughly like this: Following the use of electron microscopes to explore the structure of solids, a relation was established between the solids' macroscopic properties [...]. Thus, solid-state physics provided the theoretical foundations not only for metallurgy but also for the semiconductor and glass industries. This narrative emphasizes the coherent body of knowledge focused on structure-sensitive properties, which inspired a common approach to all classes of materials. It prompted the creation of MSE departments and a new structure of teaching undergraduate courses: to start from the first principles that connect structure and properties in all materials textbooks and then proceed to more specialized chapters on metals, ceramics, semiconductors, polymers, etc. (Ebd., S. 47).
\end{abstract}

Wenn auch die USA im Zuge der Industrialisierung weder auf Kohle noch auf Stahl verzichteten, war die wissenschaftliche Erforschung, Analyse, Optimierung und Verarbeitung entsprechender Materialien nicht an sog. Bergakademien bzw. in akademischen Forschungsprogrammen der Werkstoff- und Materialkunde organisiert (Bensaude-Vincent, 2001). Das Forschungsfeld der MSE etablierte sich in den Vereinigten Staaten als akademisches Feld erst in den 1960er Jahre als Konsequenz der Wissenschaftspolitik des Kalten Krieges (ebd.). Der Sputnik-Schock im Jahre 1957 lenkte die Aufmerksamkeit des US-amerikanischen Verteidigungsministeriums und diverser Privatfirmen auf eine verteidigungstechnische Forschungslücke: Es mangelte an Grundlagenwissen über das Verhalten von Materialien (die etwa in der Feinelektronik im Einsatz sind) unter speziellen Bedingungen wie im Weltraum oder unter radioaktiver Strahlung (Choi und Shields, 2015, S. 22). Im Rahmen des Project Pontus, einer nationalen Initiative zur Begründung der materialwissenschaftlichen Forschung in den USA, wurden mehrere Institute gegründet und an verschiedenen Hochschulen angesiedelt (ebd., S. 25). Auch wenn die Angliederung an die lokale Hochschulstruktur angepasst wurde und die Programmatiken der Institute als betont interdisziplinär kommuniziert wurden, zeichnete sich institutionenübergreifend in den USA eine gemeinsam geteilte Hierarchie im Disziplinengefüge zugunsten der physikalischen Wissenschaften ab (Mody und Choi, 2013, S. 135):

Das Feld der MSE war in den USA deutlich durch die Festkörper- bzw. Experimentalphysik dominiert, während im Gegensatz zum europäischen Trend 
die Chemie als Hilfswissenschaft der physikalischen Grundlagenforschung verhandelt wurde. Weitere beteiligte Disziplinen wie Elektrotechnik, Maschinenbau, Keramikforschung und Metallurgie galten wiederum als Anwendungsgebiete, in die sich die Kenntnisse der Grundlagenforschung transferieren lassen (BensaudeVincent, 2016, S. 48). Aus diesem Grund unterschieden sich auch die thematischen Schwerpunkte der Forschung der beiden Kontinente: In Europa dominierten in der zweiten Hälfte des 20. Jahrhunderts Forschungsprojekte, die sich primär aus chemischen Fragestellungen und Zugriffen speisten: Polymere, Keramiken, BetaAlumina, Batterieforschung und vor allem die Katalyseforschung im Bereich der Energiegewinnung, landwirtschaftlicher Anwendungen und im Dienste des Motorenbaus (Segal, 2017, S. 2ff.). In den USA hingegen zeigte sich neben dem industrienahen auch der militärische Einfluss auf die physikalisch geprägte Forschung. Neben nuklearen Technologien war das Feld lange fast synonym mit der rasant anwachsenden Halbleiterforschung in den 1960er und 1970er Jahren gesetzt worden. Siliziumprodukte wie das Si(111)-7X7, eine spezielle Oberflächenkonfiguration von Atomen in einem Siliziumkristall, revolutionierten die Schaltungen auf Computerchips und fungierten als Boundary Objects, die heterogenen Akteur/innen eine Kooperation ohne Konsens ermöglichten (Mody und Lynch, 2010, S. 4).

In diesem Zusammenspiel zwischen Experimental- und Festkörperphysik, Elektrotechnik und den aufstrebenden Informationstechnologien entstand eine vielschichtige Technoscience, die ihren Gegenstand nicht nur deskriptiv erforscht, sondern auch simultan aktiv hervorzubringen - und industriell zu vermarkten - vermag (ebd.). Als paradigmatisches Beispiel hierfür sei das Rastertunnelmikroskop (RTM) erwähnt, das als Schlüsseltechnologie in der Etablierung des USamerikanischen MSE-Paradigmas gilt (Marcovich und Shinn, 2011, S. 230). In einer Kooperation zwischen dem multinationalen IT-Konzern IBM und verschiedenen Hochschulen in der Schweiz und den USA gelang es Gerd Binnig und Heinrich Rohrer 1981, dieses technische Gerät erstmals vorzustellen, wofür sie 1986 den Physik-Nobelpreis erhielten (Binnig und Rohrer, 1986). Mit diesem Gerät lassen sich die Oberflächen von Materialien auf der innermolekularen Ebene elektronisch abtasten und durch eine Softwaresimulation visualisieren. ${ }^{4}$ Mit einem verwandten Gerät, dem Rasterkraftmikroskop, lassen sich zudem Oberflä- 
chen durch gezieltes Anbringen und Anordnen von Atomen an einer Oberfläche manipulieren (Schaper-Rinkel, 2007, S. 351).

Mit dem Rastertunnelmikroskop begann darüber hinaus eine gezielte Auseinandersetzung mit der chiralen Eigenschaft von Oberflächen, Nanopartikeln und Absorptionsprozessen von Molekülen an Oberflächen. Mit der Sichtbarmachung von "Mustern“ an Oberflächen, die sich durch die Anordnung von Atomen an einer Oberfläche bilden, entstand ein neuartiger ästhetischer Zugang zur Chiralität, der sich aus den Softwaresimulationen des Rastertunnelmikroskop speiste. In den Materialwissenschaften wurde die Chiralität bis in die späten 1990er Jahre nur sehr marginal behandelt und der zitierte Professor und Chemieingenieur versteht sich selbst als einen der Pioniere dieses Feldes, das nach wie vor nicht sehr viele Mitglieder umfasst:

Lang: Was bedeutet Chiralität speziell in Ihrer Disziplin?

Prof. Borodin: Also [Chiralität] bei den Oberflächen das war eigentlich immer ein Außenseiterthema. Als ich des angefangen habe, hab ich gedacht, ich bin der einzige auf der Welt aber das war nicht so. Aber ich hab das geglaubt und kurze Zeit später festgestellt, dass andere des auch machen aber es war da noch nicht bekannt. Und des war dann schon also 'n ganz schönes EREICNIS wenn man dann sieht, man ist jetzt nicht mal allein. Und das ist jetzt ein größeres CEBIET geworden wohl wo Leute aus verschiedenen Disziplinen das adressieren aufder NANO-Skala, nicht nur an Oberflächen, aber auch in Materialien oder in Poren oder so. [...] Und wir benutzen ein Tunnelmikroskop, das in der Schweiz erfunden wurde und das hat die Nanowissenschaften revolutioniert. Und mit dem kann man einzelne Moleküle abbilden. Submolekulare Auflösung, also ich kann keine Atome damit sehen aber ich SEHE innere Strukturen von Molekülen und wenn wirjetzt von chiralen reden, wir nehmen immer so HELIKALE Moleküle und da sehen wir dann sogar die HÄNDIGKEIT. Ich kann linke und rechte unterscheiden wenn ich das Experiment gut beherrsche was jetzt nix BESONDERES ist aber es ist schon spannend, wenn man dann bestimmte Phänomene, die mit der Händigkeit zu tun haben, untersucht. Also WIR konzentrieren uns eher aufdie zweidimensionale Kristallisation, eigentlich will ich Kristallisation verstehen - das ist so kompliziert zu verstehen, dass es kaum jemand versteht.

Die Visualisierung und Manipulation von Oberflächen und ihrer atomaren $\mathrm{Zu}$ sammensetzung ermöglichte neue epistemische wie technische Zugriffe auf die mikroskopische bzw. submolekulare Welt besonders kleiner Partikel und Oberflächenstrukturen. Sie spielte in der Erschließung der Nanoebene eine erhebliche Rolle, die seit den späten 1980er Jahren konstitutiv für die Nanowissenschaften 
war (ebd.). ${ }^{5}$ Das Feld der Nanowissenschaften überschneidet sich mit den Materialwissenschaften auf mehreren Ebenen und fungiert zugleich als soziale Arena, in der sich physikalische und chemische Konzeptionen der mikroskopischen Beschaffenheit von Festkörpern - etwa der Chiralität - in ihrer Grenzarbeit gegenüberstehen (Bensaude-Vincent, 2001). In den Materialwissenschaften ist die Oberfläche eines Materials von besonderem Interesse, da die meisten chemischen und physikalischen Prozesse an dieser ablaufen und nicht im gesamten Gefüge (der Gesamtmasse eines Werkstoffs) (Segal, 2017, S. 2). Oberflächen lassen sich dabei unter Berücksichtigung verschiedener Auflösungsebenen betrachten, wobei die Größe von Partikeln Einfluss auf deren funktionale Eigenschaften nimmt. Dies betrifft insbesondere elektrische Leitfähigkeit, optische Aktivität, katalytische Eigenschaften oder mechanische Beschaffenheit von Materialien. Nanopartikel sind über eine Größe von 1 bis 100 Nanometer definiert und die Nanowissenschaften als interdisziplinäres Feld darüber, Partikel in diesem Bereich zu erforschen und Anwendungen daraus zu entwickeln (Urban, 2015, S. 207).

Chemische und physikalische Prozesse an Oberflächen laufen je nach Größenordnung der beteiligten Strukturen unterschiedlich ab. Nanopartikel (bei Molekülen) bzw. Nanokristalle (bei Festkörpern) gehorchen beispielsweise nicht mehr den Gesetzen der klassischen Mechanik, sondern der Quantenmechanik, obwohl sie streng genommen noch immer groß genug sind, um als makroskopische Körper vorzukommen (ebd., S. 225). Es handelt sich somit um einen Grenz- bzw. Übergangsbereich zwischen der abstrakten molekularen Ebene und der makroskopischen, die sich physisch wahrnehmen lässt (ebd., S. 207). Dies zeugt davon, dass die Konzeption der Nanowelt primär durch die physikalischen Wissenschaften geprägt ist und chemische dieser als zusätzliche Perspektiven nachgeordnet sind, bzw. sich gegensätzlich zu den physikalischen verhalten. Das dem physikalischen Zugriff implizit zugrundeliegende Konzept des Materials entspricht einer ontologischen Verlängerung der Materie, unterscheidet sich maßgeblich von einem chemischen Verständnis und ist in Abgrenzung $\mathrm{zu}$ diesem konstituiert (Bensaude-Vincent, 2011, S. 114). Während die Chemie primär Nomenklaturen und Klassifikationssysteme für Materialien im Sinne einer „zoology of materials“ liefert, fügte die Physik später Kenntnisse zu Struktur, Eigenschaften und Funktionen hinzu (ebd.). Beides wird in einer berühmten Rede des Physikers Richard Feynman deutlich, in der er 1959 prophezeite, dass sich Materialien eines Tages Atom für Atom zusammensetzen lassen werden:

The principles of physics, as far as I can see, do not speak against the possibility of maneuvering things atom by atom. It is not an attempt to violate any thoden wurde erstmals auch die Chiralität von Nanopartikeln und auch als Eigenschaft des innermolekularen Aufbaus sichtbar (Gautier und Bürgi, 2009). 
laws; it is something, in principle, that can be done; but in practice, it has not been done because we are too big. Ultimately, we can do chemical synthesis. A chemist comes to us and says, "Look, I want a molecule that has the atoms arranged thus and so; make me that molecule." The chemist does a mysterious thing when he wants to make a molecule. He sees that it has got that ring, so he mixes this and that, and he shakes it, and he fiddles around. And, at the end of a difficult process, he usually does succeed in synthesizing what he wants. By the time I get my devices working, so that we can do it by physics, he will have figured out how to synthesize absolutely anything, so that this will really be useless (Feynman, 1959).

Mit diesem physikalisch geprägten Top-Down-Ansatz lassen sich Oberflächen analog zur Legobausteinmetaphorik Atom für Atom designen, aufbauen und manipulieren. Nicht zuletzt durch Technologien wie Rastertunnel- und Rasterkraftmikroskop wird es möglich, die komplexe molekulare bzw. supramolekulare Struktur auf den atomaren Aufbau zu reduzieren (Bensaude-Vincent, 2008, S. 57). Das rationale Design von Festkörpern und ihren funktionalen Eigenschaften im Sinne einer molecular manufacture wird häufig in Anlehnung an Richard Feynman gegenüber den Synthesemethoden der organischen Chemie als überlegen kommuniziert. Während klassische organische Synthesen mit Proben aus mehreren Milliarden Molekülen arbeiten und daher auf der Nanoebene chaotisch und unübersichtlich sind, kommen nanophysikalische Methoden (etwa das Rasterkraftmikroskop) i.d.R. mit einem einzigen Molekül aus, was es umweltfreundlicher und kontrollierbarer macht (Drexler, 1986, S. 13). Demgegenüber steht der chemische Bottom-Up-Ansatz, der weniger am aktiven systematischen Aufbau molekularer Maschinen ansetzt, sondern an dem Prinzip der Selbstorganisation von Molekülen und Kristallstrukturen (Urban, 2015, S. 59f.). Dementsprechend stehen sich zwei Ansätze gegenüber, wobei ersterer durch einen strengen Rationalismus des Maschineningenieurswesens und den inhärenten Drang geprägt ist, die Prozesse möglichst zu kontrollieren, überträgt letzterer die gestalterische Rolle auf die Materie selbst (Marcovich und Shinn, 2011, S 234). In diesem Sinne vertraut das Selbstorganisations-Paradigma auf den kreativen Moment der Natur und folgt der Annahme, dass sich die Einheiten in der Natur zu immer komplexer werdenden emergenten Einheiten zusammenfügen. Diese Prozesse werden auch für die Synthese und Katalyse aufgegriffen und für die Produktentwicklung genutzt, anstatt sie exante grundlegend aufbauen und rational steuern zu wollen (Schummer, 2004a, S. 17).

In der chemischen Theorie der mikroskopischen Welt verbinden sich Atome entweder zu den bereits bekannten Molekülen oder zu Kristallstrukturen. Es wird dabei zwischen Molekülen und Festkörpern unterschieden, wobei die Faustregel gilt, dass Metalle nicht-molekular, sondern kristallin sind und alle Nichtmetalle 
molekulare Strukturen bilden (Ball, 2003, S. 10f.). Da die Materialwissenschaften in ihrer Theorie- und Gegenstandsbildung erheblich durch die Auseinandersetzung mit Metallen geprägt wurden (Cahn, 2010, S. 26ff.), dient weniger der molekulare Blick der mikroskopischen Welt als erkenntnisleitend, sondern die kristalline Struktur von sog. Festkörpern. Dieser Blick unterscheidet zwischen Festkörpern und Molekülen und ist in Auseinandersetzung mit Metallen entstanden, die bei der Schmelze auf verschiedene Art und Weise auskristallisieren (Urban, 2015, S. 59). Bei der Auskühlung kristalliner Strukturen wie Metallen und Keramiken ordnen sich die Atome bzw. Partikel zu Kristallgittern in bestimmten räumlichen Konfigurationen an. Dies erfolgt durch Prozesse von Keimbildung und Kristallwachstum: Beim Abkühlen der geschmolzenen Masse setzt zu einem bestimmten Zeitpunkt der Moment ein, an dem sich spontan mehrere Atome zu einem Gitter zusammenfügen (Glazer, 2016, S. 6f.). Von diesen Kristallkeimen ausgehend fügen sich mit fallender Temperatur der Schmelze weitere Atome an, die das Muster in einer gewissen Regelmäßigkeit fortsetzen, bis eine vollständige Erstarrung eingetreten ist (ebd.). Es liegt in der Natur des Kristalls, dass die Struktur niemals vollkommen ist. Verunreinigungen bei der Keimbildung sind unvermeidbar, sodass Unregelmäßigkeiten in der Struktur auftreten, die Materialwissenschaftler/innen vor gewisse Herausforderung im Planen, Entwickeln und Skalieren von Werkstoffen stellen (Mody, 2001).

Der chemische Bottom-Up-Ansatz der molekularen Selbstorganisation wie auch der physikalische Top-Down-Ansatz des rationalen Designs unterscheiden sich dabei grundsätzlich entsprechend zugrundeliegender Prämissen und Konzeption der Materie (Schummer, 2004a, S. 9f). Der physikalische Denkstil unterscheidet sich semantisch wie epistemisch insofern vom chemischen, dass er von einer molekularen Architektur statt einer Struktur ausgeht, die sich zudem zunehmend in der Semantik der Nano- und Materialwissenschaften sedimentiert (Sierra, 2009). Moleküle werden dabei nicht als aus der Natur hervorgehende Strukturgebilde gedacht, sondern als molekulare Maschinen (Bensaude-Vincent, 2007, S. 224). Im Sinne einer mechanistischen Vorstellung von der molekularen Welt werden die Funktionsweisen makroskopischer mechanischer Systeme auf kleinstmögliche Systeme extrapoliert. Dieser Denkstil wurde zum einen von der Miniaturisierung $^{6}$ der Informationstechnologie seit den 1960er Jahren geprägt und schöpft

6 Der Begriff der Miniaturisierung der Informationstechnologie bezieht sich auf die Tendenz, dass in der Geschichte der Computersystementwicklung Prozessoren, Schaltungen und Speichermedien physisch immer kleiner und gleichzeitig immer leistungsfähiger, integrierter und vernetzter wurden und an Speicherkapazität gewannen. Als Schlagwort in diesem Zusammenhang ist „Moore's Law“ zu nennen, entsprechend dessen sich die Anzahl der Transistoren auf einem Mikrochip im Zweijahrestakt verdoppeln, während sich die Produktionskosten parallel dazu halbieren (Moore, 1965). 
sich zum anderen aus einem wachsenden Interesse von Physiker/innen an biologischen Gegenständen wie Gehirn und DNA als Informationsträger (ebd., S. 222).

Neben ihrer Rolle als Eigenschaft von Kristallbildung und -struktur ist die Chiralität in den Materialwissenschaften in zahlreiche Prozesse involviert, etwa der Katalyse, Synthese von Nanopartikeln oder Polyoxometallaten (Amabilino, 2009). Abgesehen von den kristallografischen Erkenntnissen des 19. Jahrhunderts, die zur Entdeckung der optischen Aktivität sowie zur Begründung der modernen Stereochemie beigetragen hatten und deren tragenden Rolle bei der Festlegung von Symmetrie- und Raumgruppen (Glazer, 2016, S. 7), fristete die Chiralität allerdings bis ins späte 20. Jahrhundert innerhalb der Felder der Materialforschung ein Nischendasein. Erst in den 1990er Jahren wandte sich die scientific community den räumlichen Aspekten von Materialien und Oberflächen zu (Scarso und Borsato, 2009). Dies war primär den technischen Limitationen geschuldet, denn erst durch die Rastertunnelmikroskopie wurden chirale Strukturen an Oberflächen wahrnehmbar und intelligibel. In den folgenden beiden Abschnitten wird diskutiert, welche Rolle die Chiralität als Eigenschaft von Festkörperpartikeln, Molekülstrukturen sowie fundamentalen Wechselwirkungen als Gegenstand der Boundary Work zwischen chemischen und physikalischen Kollektiven der Nanowissenschaften spielt. Die semantische Aneignung, Umdeutung, Bedeutungserweiterung und Verteidigung der konkurrierenden (und nicht immer kommensurablen) Chiralitätskonzeptionen stehen dabei im Fokus.

\subsection{Helizität, planare Chiralität und andere physikalische Zugriffe}

Die Rolle von Physiker/innen in der Materialforschung war im US-amerikanischen Kontext bis in die 1960er und in Europa bis zur Nano-Revolution der 1990er Jahre tendenziell eine randständige, wie im letzten Abschnitt hergeleitet wurde. Als Hilfswissenschaft der Chemie, die vor allem im europäischen Kontext die mentalen Modelle und Zugriffe auf das Material dominierten, waren physikalische Eigenschaften der Materie und entsprechende Methoden zwar weit verbreitet und untrennbar mit den chemischen Erkenntnisprozessen verbunden (Urban, 2015, S. 4off.). Allerdings dauerte es einige Zeit, bis Physiker/innen dezidiert erkenntnisleitende Fragestellungen und einen genuin physikalischen Zugriff auf Design, Produktion, Optimierung und Entsorgung von Werkstoffen einsetzte - und zu den Konzepten der Chemie in ein Konkurrenzverhältnis trat (Bensaude-Vincent, 2011, S. 114). Insbesondere der Ruf nach interdisziplinärer Zusammenarbeit, die Anbindungen an Elektrotechnik und Maschinenbau sowie die fortschreitende informationstechnologische Automatisierung der Forschungs- und Entwicklungsprozesse führten seit den 1960er Jahren zu einer Aufwertung physikalischer Epis- 
temiken und Methoden in hybriden und anwendungsorientierten Bereichen wie den Nano- und Materialwissenschaften (Mody und Choi, 2013).

Dass die Denkstile der Festkörperphysik in den Material- und Nanowissenschaften zunehmend dominant werden und diejenigen der Chemie allmählich verdrängen, zeigt sich in den entsprechenden feldspezifischen Narrativen zur (supra)molekularen Welt: etablierte chemische Konzeptionen des Molekularen wie etwa die Konzeption der molekularen Chiralität - erfahren eine sukzessive Bedeutungsverschiebung. Im Gegensatz zu den in Kapitel 4 und 5 behandelten Life Sciences, in denen ein biowissenschaftlicher Zugriff auf das Molekulare chemische Konzepte aus der Terminologie und Forschungspraxis verdrängt und unsichtbar werden lässt, zeigt sich für das Feld der Materialwissenschaften, dass der Chiralitätsbegriff dort vielmehr angeeignet und erweitert wird. Als Chemiker/innen sozialisierte Personen im Feld zeigen sich angesichts der Beobachtung besorgt, dass auch solche Phänomene neuerdings als chiral bezeichnet werden, die im „strengen Sinne“ nicht chiral sind. Als Gegenstand der Grenzarbeit gegen den wachsenden physikalischen Einfluss in ihrem Feld wird die Chiralität als genuin chemischer Gegenstand verteidigt. Die folgenden Beobachtungen aus dem Feld zeigen, wie ambivalent sich das Verhältnis zwischen Chemie und Physik auf der experimentellen Ebene gestaltet: Estyleinerseits wirken ungeschriebene Präideen einer disziplinären Hierarchie auf die Narrativbildung ein und andererseits zeigen sich erhebliche Inkommensurabilitäten bei den grundlegenden geteilten Konzeptionen des Molekularen.

In diesem Abschnitt werden zunächst die physikalischen Perspektiven auf den Gegenstand des Materials am Fallbeispiel der Chiralität bzw. der Helizität referiert. Es zeigt sich, dass in Bezug auf etablierte chemische Konzepte der mikroskopischen Welt eher der Modus einer Aneignung stattfindet, sodass diese in ihren epistemischen Bezügen, Reichweiten und Bedeutungen eine Verschiebung erfahren. In Abschnitt 3.3 wird anschließend aufgezeigt, wie Chemiker/innen im Feld auf die Aneignung von Begriffen, Methoden und Forschungsgegenständen vonseiten der Physik reagieren, indem der physikalischen Begriffserweiterung durch definitorische Einschränkungsversuche entgegengewirkt wird.

Lang: Was verstehen Sie unter Chiralität? Dr. Eller: (1) Chiralität, ja das ist (4) also erstmal ein Konzept, das ich (1) eben jetzt (2) aufgrund meiner (2) spezialisierten Ausbildung einfach aus der Elementarteilchenphysik vorwiegend kenne. Da spricht man auch von Linkshändigkeit und Rechtshändigkeit oder auch von Helizität (.) und CHIRALITÄT, das sind glaub ich einfach Synonyme. (5) Und man sagt eben dann (7) also es gibt in der Teilchenphysik dieses Konzept der (.) Links-und Rechtshändigkeit, das besagt ähm (1) eben einfach ein Elementarteilchen hat 'nen SPIN (2) also üblicherweise 'nen halbzahligen Spin. [...] 
Werden Physiker/innen verschiedener Fachrichtungen nach ihrem Chiralitätsverständnis befragt, verwenden diese das Konzept häufig synonym mit bzw. setzen es in ein Abhängigkeitsverhältnis von der Helizität, einer originär quantenmechanischen Konzeption. Physikalische und chemische Definitionen der Chiralität unterscheiden sich grundlegend in Bezug auf den jeweiligen Phänomenbereich. Während sich die chemische Chiralität weitestgehend auf die inhärente Spiegelasymmetrie molekularer bzw. supramolekularer Strukturen bezieht, bewegt sich das physikalische Konzept auf der Ebene von Elementarteilchen und fundamentalen Wechselwirkungen der Materie (siehe hierzu auch Kapitel 6). In der weiteren Betrachtung wird deutlich, dass diese quantenphysikalischen Konzeptionen mittlerweile in den interdisziplinären Feldern der Material- und Nanowissenschaften omnipräsent sind und sich mit den chemischen vermengt haben. Insbesondere der Begriff der Helizität, die ursprünglich als reine Erhaltungsgröße der Quantenfeldtheorie konzipiert war, erfährt in diesem Kontext eine erweiterte Rezeption in Bezug auf Gegenstände supramolekularer Strukturen. Dabei verdrängt der physikalisch besetzte Begriff der Helizität allmählich den chemischen der Chiralität und die Distinktion zwischen den beiden Konzeptionen wird kontinuierlich aufgeweicht.

Der Begriff der Helizität im Sinne der Quantenfeldtheorie bezeichnet diejenige Eigenschaft eines Teilchens, die dem Spin des Teilchens den entsprechenden Impuls gibt und darüber entscheidet, in welche Richtung es entsprechend rotiert (Philipsen, 2018, S. 57f.). Makroskopisch gesprochen muss man sich die Bewegung des Teilchens so vorstellen, dass es eine rechts- bzw. linksgängige Schraubenlinie nach vorne (rechts) bzw. hinten (links) beschreibt. Es handelt sich allerdings bei den quantenmechanischen Konzeptionen von Helizität und Chiralität nicht um molekulare oder andere makroskopische Strukturen der Materie, sondern um mathematische Abstraktionen der Teilchendynamik (vgl. Kapitel 6). Der Begriff der Helizität wird allerdings nicht nur in der Teilchenphysik verwendet, sondern seit den 1980er Jahren auch in den Nano- und Materialwissenschaften (Scarso und Borsato, 2009). In diesem Bereich setzt er sich seither sukzessive gegen das organisch-chemische Konzept der axialen Chiralität durch und die beiden Definitionsbereiche verschwimmen zunehmends ineinander (Ernst, 2012, S. 261). Bei helikalen bzw. axial-chiralen Molekülen handelt es sich i.d.R. um längere, kettenförmige Moleküle wie etwa Polymere, die in ihrem regelmäßigen Aufbau eine dreidimensionale Schraubenform aufweisen (vgl. Abb. 3.1). Das wohl prägnanteste Beispiel für eine axiale molekulare Chiralität bietet in diesem Zusammenhang die DNA-Doppelhelix mit ihrer charakteristischen Schraubenform (Sierra, 2009). Die Stereochemie verwendet für diese Art der Chiralität eine eigene Nomenklatur, um sie von der planaren Chiralität kleiner, optisch aktiver Substanzen abzugrenzen. Angaben zur Windungsrichtung werden mit (P)-(+) (plus) für im Uhrzeigersinn und mit (M)-(-) (minus) für gegen den Uhrzeigersinn angegeben (vgl. Abb. 3.2). 


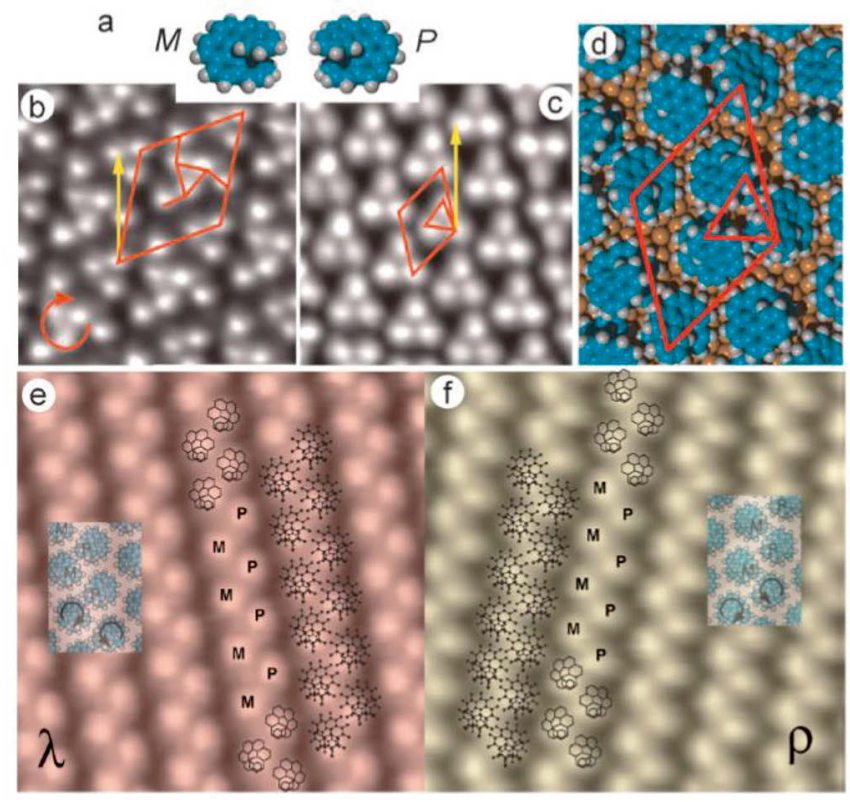

Bei dieser Abbildung zweier gegensätzlicher helikaler Schraubenmoleküle handelt es sich um eine softwaregestützte Simulation, die auf einer Rastertunnelmikroskopie beruht. Es handelt sich dabei um chirale Kohlenwasserstoffe, die zunächst nach Enantiomeren getrennt und anschließend auf einer Cu(111)-Oberfläche absorbiert wurden. Entsprechend entstand ein axiales Muster. Entnommen aus (Ernst, 2012, S. 2072).

(a)

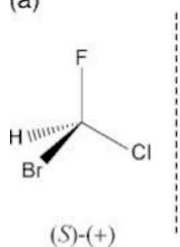

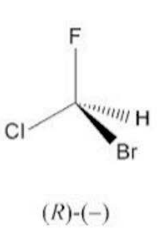

(b)

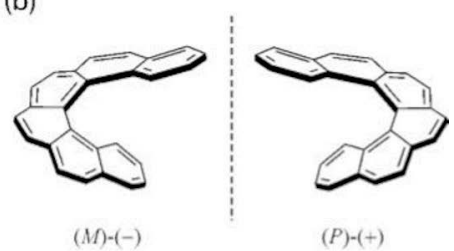

Die Nomenklaturen organischer Verbindungen mit einem Stereozentrum und helikaler Supramoleküle wie dem Hexahelicen werden in der Stereochemie voneinander abgegrenzt. Abbildung übernommen aus (Barron, 2009, S. 3).

Die Helizität als Sonderform der supramolekularen Chiralität gewann in den 1990er Jahren allmählich an Bedeutung, als sie zu einem essentiellen Gegenstand der (chemisch ausgerichteten) Materialwissenschaften avancierte (Rickhaus et al., 2014). Dies liegt darin begründet, dass die molekulare Selbstorganisation an Ober- 
flächen häufig chirale oder schraubenförmige molekularen Strukturen hervorbringt. ${ }^{7}$ Diese Oberflächenstrukturen können wiederum auf verschiedene Prozesse wie die enantioselektive Katalyseleistung Einfluss nehmen, worauf in diesem Zusammenhang im darauffolgenden Abschnitt 3.3 näher eingegangen wird. Im Gegensatz zur Chiralität wird am Begriff der Helizität deutlich, dass dieses Konzept in den Nano- und Materialwissenschaften als tendenziell eher physikalisches Prinzip wahrgenommen wird und die Grenzen zwischen epistemischen Bezügen auf Elementarteilchendynamiken, Nanopartikel sowie molekulare und supramolekulare Gebilde unscharf werden.

Lang: Welche wissenschaftlichen Fragestellungen finden Sie im Zusammenhang mit Chiralität besonders interessant und relevant? Chemieingenieur Prof. Borodin: Einmal schon noch Material wissenschaften, irgendwelche optischen Schalter in der Richtung. (1) Wenn man also [ein] Material macht, das dann sozusagen als SENSOR oder als Schalter nützlich ist (2) und dann natürlich nach wie vor die Paritätsverletzung und der Einfluss auf das Universum - da spielt's eine Rolle. Und für das LEBEN oder nicht? Das wird ja immer diskutiert.

Im hybriden disziplinären Grenzbereich der Oberflächen-, Nano- und Materialforschung lässt sich zunehmend beobachten, dass die Chiralität nicht mehr als genuin chemisches Konzept verstanden wird, sondern dass Physiker/innen in ihren Semantiken eine ebenbürtige Expertise für sich beanspruchen. Die Interviewstudie zeigt im Vergleich, dass relativierende Aussagen wie „Ich bin kein Chemiker, von daher kann ich nicht allzu viel dazu sagen" deutlich seltener ins Feld geführt werden als etwa von Lebenswissenschaftler/innen und vor allem Ärzt/innen. Physiker/innen positionieren sich selbstbewusst als Wissensproduzent/innen im Bereich chiraler Moleküle und deren experimenteller Manipulation, ein Feld, das bislang Vertreter/innen der organischen Chemie vorbehalten war. Die physikalische Auseinandersetzung beschränkt sich nicht auf die epistemische Aneignung dieses molekularen Phänomens. Vielmehr bedeutet die Entgrenzung physikalischer Zugriffe auf die molekulare Welt eine erhebliche Erweiterung des Chiralitätsbegriffes, denn ein Zugriff auf die molekulare Architektur von chiralen Oberflächen kann nicht mehr erfolgen, ohne auf die prozesssteuernden Grundkräfte und die physikalische Konzeption der Nanopartikel einzugehen. Die supramolekulare Welt erweist sich als Konglomerat verschiedener disziplinärer Zugriffe (auch aus den angewandten Ingenieurswissenschaften) mit einer gewissen Diskurshegemonie der Physik ${ }^{8}$ als vermittelnde Instanz.

Die Ursachen und Mechanismen der molekularen Selbstorganisation gelten nach wie vor weitestgehend als rätselhaft, insbesondere da sich achirale Nanopartikel und Moleküle zu chiralen Makromolekülen zusammenschließen können (Ernst, 2012, S. 2061). 


\begin{abstract}
Eperimentalphysiker Dr. Schoeck: Also ich hab' [in meiner Doktorarbeit] zumindest mit den Chemikern zusammengearbeitet, die unsere Moleküle gemacht haben. Und [mit den] Leute [n], die des Canze simuliert haben, das [machen] bei uns die theoretischen Physiker. Da war's für mich auch interdisziplinär weil's halt was anderes war als mich einfach nur hinzusetzen und irgendwelche Sachen zu messen. Und ich bin sogar mal ab und zu zu den Mathematikern rübergelaufen und hab mich mit denen einfach über Symmetrie unterhalten.
\end{abstract}

In der Wissenschaftsforschung herrscht Uneinigkeit darüber, ob es sich bei den den Nanowissenschaften tatsächlich um neuartige Felder mit eigenständigen Phänomenen und Methoden handelt (Marcovich und Shinn, 2011, S. 221) oder eher um eine Verlängerung der Festkörper- und Halbleiterphysik bzw. der Kolloidchemie unter dem Vorzeichen neuerer wissenschaftspolitischer Dispositive (Schummer, 2004a). Auf der Ebene von impliziten Konzeptionen des Molekularen zeigt sich, dass hier tatsächlich ein neuartiger, dezidiert interdisziplinärer, Zugriff entstanden ist, der sich deutlich von den organisch-chemischen, anorganisch-chemischen und physikalischen abgrenzen lässt. Die Hegemonie der physikalischen Zugriffe, im Rahmen derer etablierte chemische Konzepte aneignet und diese um eigene Perspektiven erweitert werden, wird darüber hinaus durch entsprechende Dispositive der Forschungsförderung mitgetragen (Mody und Choi, 2013). Die implizite Bevorzugung eines physikalischen style of reasoning zeigt sich etwa darin, dass physikalische Mikroskopiemethoden der klassischen organischen Synthesechemie gegenüber als überlegen eingeschätzt werden und entsprechend eine gesteigerte Förderung in der Nano- und Materialforschung erfahren (Bensaude-Vincent, 2008, S. 58). In den Innovationsdiskursen der Oberflächen-, Nano- und Materialforschung ließ sich eine besonders prägnante und wirkmächtige Präidee herausarbeiten, die die physikalische Hegemonie stützt: Die Präidee des Reduktionismus wird in der Folge vorgestellt und in Bezug auf die Konzeption der Chiralität und der supramolekularen Welt diskutiert.

Die Grundannahme, dass chemische Gegenstände grundsätzlich mit physikalischen Größen, Kräften und Gesetzen erklärbar bzw. auf diese zurückzuführen seien, wird im Folgenden als Reduktionsthese bezeichnet und in Kapitel 6 vertiefend diskutiert. Die Reduktionsthese, die seit der erfolgreichen Etablierung der Quantentheorie in der wissenschaftsphilosophischen Schule des Wiener Kreises ausformuliert worden war, ist trotz Jahrzehnten der Kritik nach wie vor im allgemeinen Wissenschaftsverständnis vieler Fachwissenschaftler/innen fest verankert (Janich, 1998). Als unhinterfragte Wahrheit lebt die Präidee einer Hierarchie der Wissenschaften, die die physikalischen Grundlagenwissenschaften privilegiert, auch in den Innovationsdispositiven der Forschungsförderung fort (vgl. 5.2). Die Nanowissenschaften sind nicht zuletzt deshalb so erfolgreich in der Generierung von Ressourcen, weil sich diese Präideen der Einheit und Hierarchie von 
Wirklichkeitsebenen und wissenschaftlichen Disziplinen in einem der zentralen Gegenstände dieser hybriden Felder wiederfinden: der molekularen Selbstorganisation. Die „molecular self-assembly“ stellt gewissermaßen den kleinsten gemeinsamen Nenner dar, der das sonst eher heterogene, interdisziplinäre Feld mit eher lokalen Wissenssilos miteinander verbindet (Marcovich und Shinn, 2011, S. 236).

Dr. Schoeck: Woran ich [bei Chiralität] auch noch denken muss, ist an meine selbstorganisierten Moleküle und da ist Chiralität eines der Organisationsprinzipien, die dahinter stecken. Das bedeutet (.) eine Form über die sich Moleküle organisieren können, ist zum Beispiel über irgendwelche Form von KRÄFTEN also elektrostatische Kräfte zum Beispiel ähm ordnen sie sich zu Einheiten AN (.) aber auch je nachdem welche Händigkeit sie haben ordnen sie sich-also (1) dass sie manchmal so kleine Einheiten bilden. Diese kleineren Einheiten bilden größere Einheiten die bilden wieder größere Einheiten und diese bilden wieder größere Einheiten. Einer dieser SCHRITTE wird zum Beispiel von Chiralität bestimmt, da muss ich dran denken.

Die Vorstellung einer Natur als System, das sich in unterschiedliche Komplexitätsgrade der Betrachtung (fundamentale Wechselwirkungen, Elementarteilchen, Atome, Moleküle, Makro- und Supramoleküle, Zellen, Organismen, Populationen) aufteilen lässt, wird in diesem Kontext besonders schlüssig (Bensaude-Vincent, 2016, S. 48). Die Präidee der Hierarchie der Wissenschaften bewegt sich parallel dazu: Entsprechend ihres jeweiligen epistemischen Zuständigkeitsbereiches ordnen sich die Disziplinen in einer vertikalen, scheinbar naturgegebenen Hierarchie an (Folkers, 2011). Das Phänomen der molekularen Selbstorganisation erweist sich in der Formulierung des zitierten Physikers Dr. Schoeck als paradigmatischer Fall für ein emergentes ${ }^{9}$ System: Atome und Moleküle lagern sich unter der Einwirkung physikalischer Einflüsse von Zeit-, Ladungs- und Paritätsinvarianzen zu spezifischen Mustern ab bzw. lassen sich gar dahingehend steuern. Die Reduzierbarkeit molekularer Phänomene auf die fundamentalen Wechselwirkungen der Materie erweist sich zudem in kaum einem anderen Bereich als derart evident wie im Falle molekularer Selbstorganisation an Oberflächen: Gerade bei Nanopartikeln wird die Schnittstelle bzw. der Übergangsbereich zwischen klassischen und quantenmechanischen Gesetzen materiell erfahrbar (Urban, 2015, S. 253). Die implizit angenommene Hierarchie von fundamentalen Wechselwirkungen, Elementarteilchen, Atomen, Nanopartikeln, Molekülen und Oberflächen (und den entsprechenden wissenschaftlichen Disziplinen) wird darüber hinaus durch industrielle Innovationen im Bereich der MSE reproduziert und legitimiert (Bensaude-Vincent, 2007, S. 229): 
Die Nano- und Materialwissenschaften erwiesen sich als sehr anpassungsfähig gegenüber teilweise dramatisch ausfallenden Paradigmenwechseln in der US-amerikanischen wie europäischen Forschungsförderung des 20 . Jahrhunderts. Obwohl das der Grundlagenforschung zugewandte Innovationsmodell des Kalten Krieges, das mit dem anwendungs- und relevanzorientierten Paradigma der 1960er und 1970er Jahre keinen schärferen Kontrast bilden könnte, gingen diese Forschungsbereiche keinesfalls mit dem entsprechenden Paradigmenwechsel unter (Mody und Choi, 2013, S. 125). Auch mit den neueren Innovationsdispositive im Sinne einer "commercialized postmodern university“, die sich vor allem durch einen unternehmerischen Innovationsgedanken auszeichnet, geraten die hybriden Forschungsbereiche nicht in Konflikt (ebd.). Der Chemiehistorikerin Bernadette Bensaude-Vincent zufolge fungiert insbesondere der vage und offene Materialbzw. Werkstoffbegriff als „boundary category, which helps to create a community of practitioners", sodass in einem hybriden und heterogenen Forschungsfeld Kooperation ohne Konsens möglich ist (dies. 2011, S. 117). Der Erfolg der beiden hybriden Forschungsbereiche seit den 1990er Jahren ist darüber hinaus darauf zurückzuführen, dass die zugrundeliegenden Präideen mit den Innovationsdispositiven neoliberaler Big Science-Förderung übereinstimmen:

There is a popular view of the sciences, according to which a hierarchy of material objects is mirrored by a hierarchy of the disciplines: the basic science (called physics) deals with the smallest objects, elementary particles or atoms, that are the building blocks of the objects of the next level, namely molecules which define the field of chemistry. Next comes biology that deals with living beings that are made up of molecules, and finally, if you wish, sociology. Not surprisingly, that originally pre-modern view found expression in the 19th century, when the rapid formation and differentiation of scientific disciplines broke up old dreams of the unity of science. No doubt, creating a new unity of the sciences by conceiving a division of labor according to the scale of their objects served as a sedative for those who wished to hold on to such unity (Schummer, 2004a, S. 15).

Die Innovationspolitik der Converging Technologies, die in den USA um die Jahrtausendwende ihren Anfang nahm, äußerte bezüglich einer multidisziplinären Zusammenarbeit (Konvergenz) von Nano-, Bio- und Informationstechnologie sowie Kognitionswissenschaften (NBIC) zweierlei positive Erwartungen: Zum einen würden diese ein ganzheitliches wissenschaftliches Verständnis komplexer Gegenstände wie etwa dem menschlichen Gehirn oder dem Klimawandel fördern und zum anderen Innovationen schaffen, die ebenso komplexe gesellschaftliche Bedarfslagen befriedigen würden (Bensaude-Vincent, 2016, S. 51f.). Zu letzterem zählen etwa energieeffiziente Hochleistungsmaterialien oder maßgeschneiderte Arzneistoffe für komplexe Krankheitsbilder wie etwa Morbus Alzheimer oder 
Krebs (siehe Kapitel 4). Die Prämissen der NBIC-Programmatik beruhen dabei auf den Prämissen der Wissenschaftsphilosophie des Reduktionismus und der Einheitswissenschaft in der Spielart des Wiener Kreises (ebd.). Die Idee der Einheitswissenschaft basiert auf der Annahme einer Hierarchie der Wissenschaften, die in einem harmonischen, ganzheitlichen Erkenntnisprozess Wissen entlang von naturgegebenen Betrachtungsebenen austauschen. Diese Präideen galten in der Wissenschaftsphilosophie lange als überholt, erfuhren jedoch mit dem Aufkommen des NBIC-Paradigmas eine Renaissance (Schaper-Rinkel, 2007). Das Kernkonzept der verschiedenen Reduktions- bzw. Emergenzansätze - die Betrachtungsebene - erfuhr mit dem Siegeszug der nano scale research eine besondere Aufwertung.

Die mikroskopischen Welten der Nano- bzw. der supramolekularen Ebene wurden in einem sozio-technischen Netzwerk verschiedener Technologien, wissenschaftlicher Disziplinen, Institutionen, Publikationen, wissenschaftspolitischen Institutionen, etc. hervorgebracht. Als boundary categories ermöglichen diese Betrachtungsebenen nicht nur die Kooperationen unter heterogenen Akteur/innen, die ansonsten wenige Prämissen, epistemische Weltbilder und methodische Herangehensweisen teilen. Vielmehr werden diese neu hervorgebrachten epistemischen Räume dadurch aufrechterhalten, reifiziert und bestätigt. Die fortschreitende Etablierung eigenständiger Forschungsdisziplinen wie Nano and Materials Sciences, die aufgrund eigenständiger Methoden, Gegenstände, Theorien und Fragestellungen sich von ihren Mutterdisziplinen abgrenzen, wirkt sich zudem positiv auf die Festigung der Betrachtungsebene als erkenntnisleitende Präidee des NBIC-Paradigmas aus (Marcovich und Shinn, 2011). Die Präideen von Einheitswissenschaft und Hierarchie der Wissenschaften, wie sie mit den Converging Technologies wiederaufleben durften, privilegieren seit jeher die physikalischen Wissenschaften als Königsdisziplin, was sich auch in den Nano- und Materialwissenschaften am Fallbeispiel der Chiralität beobachten lässt.

Die Chiralität wird in den jüngeren Diskursen der Wissenschaftsphilosophie verstärkt als Phänomen gerahmt, das als Universalismus der Natur die Übersetzung zwischen ontologisch gefassten Betrachtungsebenen ermöglicht (vgl. Abschnitt 6.2). Die stereoselektive molekulare Selbstorganisation gilt neben anderen chiralen Gegenständen (chirale Nanopartikel) und Prozessen (enantioselektive Biokatalyse), die sich genuin auf der Nano- bzw. supramolekularen Ebene beobachten lassen, als eindrückliches Beispiel. Es lassen sich nämlich die Übergangszustände der Materie nachvollziehen, insbesondere zwischen subatomarer, atomarer und molekularer Betrachtungsebene. Die Möglichkeit einer direkten Rückführbarkeit makroskopischer Vorgänge der Materie auf die fundamentalen Gesetze und Kräfte der Physik entspricht der physikalistischen Ideenwelt der Reduktions- bzw. Emergenzparadigmata, die den Traum der Einheit der Wis- 
senschaft und einer sog. Entschlüsselung des Buches der Natur noch nicht aufgegeben haben. Die Chiralität erfährt jedenfalls in diesem Zusammenhang eine erhebliche Verschiebung ihrer Bedeutungszusammenhänge und epistemischen Bezugnahmen. Das ehemals genuin organisch-chemische Konzept wird zunehmend von den physikalischen Wissenschaften vereinnahmt und in seinen epistemischen wie ontischen Bezügen ausgeweitet. Im folgenden Abschnitt werden die Reaktionen aus den Feldern der Chemie auf diese Sachverhalte geschildert.

\subsection{Chiralität im „strengen Sinne”}

Für viele Chemiker/innen, die in den Feldern der Material-, Nano- und Oberflächenforschung beschäftigt sind, gehören ein regelmäßiger Austausch und Projektkooperationen mit Physiker/innen zum Alltag. Die verschiedenen chemischen wie physikalischen Fachbereiche erweisen sich als derart amalgamiert, dass kaum eine klare Distinktion mehr möglich ist, „wo die Chemie aufhört und die Physik bereits anfängt“. Diese und andere alltägliche Formulierungen wie „wir machen hier eigentlich schon Physik" werden in den beobachteten Feldern der anorganisch-chemischen Materialforschung und den entsprechenden Interviews häufig geäußert. Die Nähe und Überschneidung zwischen chemischen und physikalischen Gegenstandsbereichen sorgt aus diesem Grund für eine ambivalente Semantik. Einerseits, so zeigt sich, neigen Chemiker/innen zu einer Adaption physikalischer Zugriffe auf die supramolekulare Welt. Nicht nur physikalische Gerätschaften wie das Rastertunnelmikroskop und quantenphysikalische Erklärungen der molekularen Prozesse, sondern auch der in Abschnitt 6.2 ausgeführte Schilderungsduktus zur Chiralität wird als Universalismus übernommen. Demnach wird die Chiralität ähnlich wie in den entsprechenden Diskursen des neueren Emergenzparadigmas als rarer Universalismus der Natur kommuniziert, bei dem sich Kräfte- und Teilchendynamiken sowie anorganische wie organische molekulare Strukturen, die molekularen Bausteine des Lebens und komplexe makroskopische Systeme wie Organismen, Gehirne und Sozialverhalten positiv zu einer einheitlichen Wirklichkeit der Natur aufschichten.

Chemiker/innen in diesen hybriden Feldern übernehmen diese Narrative, Epistemiken, Weltbilder, Präideen und richten auch ihr wissenschaftliches Handeln danach aus. Die Reduzierbarkeitsthese chemischer auf physikalische Theorien und Gesetze löst keinesfalls Unbehagen aus, sondern wird vielmehr als Legitimationsquelle für die eigene hochstehende Position im hierarchischen Gefüge der Naturwissenschaften angeführt. Auf der anderen Seite sind Chemiker/innen sich des wachsenden Einflusses physikalischer Zugriffe auf die chemische Fachsprache, Methoden und Gegenstände bewusst, was zu Identitätskonflikten und Abgrenzungsbestrebungen führt. Im Alltag äußern entsprechende 
Akteur/innen, dass in den letzten Jahren die Experimentalphysik sich vermehrt den Molekülen annimmt und sich die molekulare Welt - etwa das Konzept der Chiralität - aneignet, mit physikalischen Konzeptionen überformt und von ihrem chemischen Ursprung entfremdet. Die Abwehr falscher Konzeptionen der molekularen Welt und der Chiralität im Speziellen zeigt sich etwa in den häufigen Historisierungen dieser Phänomenbereiche. So bemühen sich Chemiker/innen darum, zu betonen, dass bestimmte Gegenstände von Chemiker/innen entdeckt wurden, die damit einen enormen Beitrag zum menschlichen Fortschritt geleistet haben. Dieser Schilderungsduktus verhandelt implizit wie explizit, dass die Errungenschaften der Chemie gegenwärtig von Physiker/innen angeeignet und damit unsichtbar gemacht werden. Diese Narrative der Grenzarbeit seien in der Folge wiedergegeben.

Chemieprofessorin Pejačević: Ich bin mehrheitlich fast GRENZgänger, also ich hab in meiner Ausbildung auf dem Papier, in meiner venia legendi die Habilitation in anorganischer Chemie erhalten. Aber es ist dann mehr Materialwissenschaften geworden. Ich war'ne Zeit lang SEHR STARK ausgerichtet aufNANO als ich so am Anfang meiner eigenen Karriere stand und dann hat sich's mehr und mehr hinentwickelt zu [Katalyseforschung]. Und das ist natürlich ein umbrella, der auch nicht nur klassische Anorganik oder klassische Nano oder Materialforschung, sondern eigentlich ALLE Bereiche molekularer und auch [lacht] der Nanochemie war.

Wie bereits im ersten Abschnitt dieses Kapitels ausführlich dargelegt wurde, sind die wissenschaftlichen Disziplinen von Chemie und Physik seit den 1980er Jahren im Bereich der Materialwissenschaften sowie der Nano- und Oberflächenforschung verstärkt zusammengewachsen, was sich auch in der Fachidentität der beteiligten Akteur/innen niederschlägt. Während bis dahin physikalische wie chemische Zugriffe auf das Material sich erheblich voneinander unterschieden hatten, bilden sie mittlerweile einen hybriden disziplinären Bereich, in dem Grenzziehungen kaum noch intelligibel und praktikabel sind (Bensaude-Vincent, 2016). Seit dem 18. Jahrhundert etablierte sich die Chemie beinahe exklusiv als die rationale Wissenschaft von den Materialien, was sich in der zweiten Hälfte des 20. Jahrhunderts änderte, als im Zuge des Kalten Krieges die physikalischen Wissenschaften ergiebige Anwendungsgebiete insbesondere mit dem Aufkommen der Computertechnik- und Raumfahrtindustrie erschließen konnten (Hentschel, 2011). Insbesondere in den USA avancierte die Chemie zur Hilfswissenschaft der physikalischen Leitdisziplin, was allerdings in der Wahrnehmung der in der Materialforschung beschäftigten Chemiker/innen keinesfalls negativ bewertet wird. Demnach profitierte die Chemie explizit von der Amalgamierung mit der Physik, insbesondere durch die Adaption dezidiert physikalischer Methoden wie der in Abschnitt 6.1 vorgestellten Rastertunnelmikroskopie. Ohne diese wäre chemisches 
Wahrnehmen und Forschen in der nanoskopischen und supramolekularen Welt von Materialien, Oberflächen und Partikeln nicht möglich.

Auch von den Innovationsdispositiven des NBIC-Paradigmas wussten Chemiker/innen zu profitieren, indem sie den Forderungen nach Interdisziplinarität, Großprojektforschung, Industrienähe und gesellschaftlicher Relevanz ihrer Forschung entsprechend die Zusammenarbeit mit Physiker/innen (sowie Biolog/innen und Vertreter/innen der verschiedenen Ingenieurswissenschaften) in diesem Bereich suchten (Urban, 2015, S. 265f.). Im Zuge dessen zeigt sich, dass auch das grundlegende Verständnis des Molekularen und seiner zentralen Konzepte unter Chemiker/innen in diesen Bereichen ein anderes geworden ist. Als besonders eindrückliches Beispiel hierfür dient die Beobachtung, dass insbesondere im Bereich der Nanoforschung das mentale Modell der Chemiker/innen vom dreidimensionalen Modell wieder abrückt und auf ein 2D-Modell zurückkehrt (Atkins, 2015, S. 90). Während in der Historiografie der Chemie die Erschließung der räumlichen molekularen Struktur zu den Sternstunden der Disziplin zählt (vgl. Abschnitt 2.1), scheint diese in den gegenwärtigen Grenzbereichen der Chemie wieder rückgängig gemacht zu werden. "Chemistry's migration from three to two dimensions“, die jüngst beobachtet wird, wird in den Zusammenhang mit der Entdeckung des Graphens gesetzt (ebd.). ${ }^{10}$ Graphen bildet die Ausgangsstruktur für weitere vielversprechende Materialstrukturen wie Fullerene und Kohlenstoffnanoröhren, die sich wiederum vielfältig in der Produktentwicklung und -optimierung einsetzen lassen (Bensaude-Vincent, 2007).

Die Renaissance der zweidimensionalen Betrachtung materieller Mikrostrukturen steht dabei in Zusammenhang mit dem Fokus auf die Oberfläche von Materialien, die, wie bereits angeführt, in den Materialwissenschaften besondere Aufmerksamkeit genießt. Da die meisten chemischen wie physikalischen Prozesse (wie etwa die Katalyse) an der Oberfläche von Stoffen stattfindet, setzte sich analog zur betrachteten Fläche ein planares mentales Modell dieser Prozesse durch (Segal, 2017, S. 2). Auch die Chiralität - entweder als Eigenschaft von molekularen Gebilden selbst oder im Kontext von enantioselektiven Absorptions- oder Katalyseprozessen an Oberflächen (Barron, 2009) - verliert dabei sukzessive ihren exklusiv dreidimensionalen Charakter, ohne den sie bislang kaum intelligibel erschien. Vielmehr etabliert sich in den Nano- und Oberflächenwissenschaften an

10 Beim Graphen handelt es sich um ein besonders vielversprechendes, da außerordentlich stabiles Material, das über besondere Eigenschaften verfügt und zahlreiche technische Innovationen verspricht. Strukturell gesprochen handelt es sich um eine Modifikation des Kohlenstoffs, bei dem sich C-Atome in zweidimensionalen Bienenwaben- bzw. Hühnerdrahtmustern anordnen (Loeve und Bensaude-Vincent, 2017). Diese zeichnen sich durch eine sechseckige Struktur aus, ähnlich wie der aus Abschnitt 2.1 bekannte Benzolring, allerdings in 2D. 
den hybriden Grenzen von chemischen und physikalischen Zugriffen auf die mikroskopische Welt ein Konzept der planaren Chiralität, was durchaus umstritten ist:

Unfortunately, with chirality becoming part of surface science the term „2D chirality" is often used nowadays. Planar molecules aligned into the plane (i.e., $2 \mathrm{D}$ confinement) by a surface or a physical field still suffer a polarization of their electron cloud perpendicular to that plane, giving it an up and a down; so it is a truly three-dimensional (3D) object. It goes without saying that when surface atoms are also taken into account, i.e., when the adsorbate as a whole is considered (as it always should), we deal with a 3D chiral entity. Although scholarly discussions on chirality in different dimensions can be quite useful, 2D chirality remains a purely theoretical construct. For real experimental systems, this term is inappropriate and misleading (Ernst, 2012, S. 261).

Die Auseinandersetzungen mit den konzeptionellen Aneignungen seitens der Physik zeigen darüber hinaus, dass die Grenzarbeit der chemischen Felder nicht nur gegen außen erfolgt, sondern auch unter den chemischen Subdisziplinen selbst. Am Beispiel der Nano- und Oberflächenforschung zeigt sich, dass Chemiker/innen, die in diesen hybriden Bereichen beschäftigt sind, eine deutlich größere Nähe $\mathrm{zu}$ den physikalischen Theorien, Methoden und styles of reasoning ${ }^{11} \mathrm{kom}$ munizieren als etwa $\mathrm{zu}$ den Feldern der organischen Chemie und Biochemie. Im Bereich Nano- und Materialwissenschaften bewegen sich die Vertreter/innen der Chemie mit ihren Aktivitäten überwiegend in der physikalischen Chemie sowie Elektrochemie, Kristallografie und technischen Werkstoffkunde und stehen inhaltlich wie methodisch in einer deutlich engeren Beziehung zu den physikalischen Feldern als zu denjenigen der klassischen organischen Synthesechemie. Sie sind überwiegend in der anorganischen Chemie sozialisiert und/oder spezialisiert und markieren eine Grenze zur organischen Chemie anhand der strikten Distinktion „we do solid state chemistry, we don't deal with molecules“, wie es eine Dokto-

Das analytische Konzept der styles of reasoning (Hacking, 1992) orientiert sich an Ludwik Flecks Theorie der Denkstile und Denkkollektive (Fleck, 2012) und findet vor allem in wissenschaftshistorischen und -philosophischen Kontexten Anwendung. Styles of reasoning beziehen sich auf den intellektuellen Prozess der wissenschaftlichen Generierung von Evidenz, der nicht etwa als rein objektiven, singulären Heureka-Moment verstanden wird, sondern als Ergebnis interferierender kollektiver Auseinandersetzungen mit einen Gegenstand. Demnach treten die Stile aufgrund unterschiedlicher Präideen, soziotechnischen Settings, Modi der wissenschaftlichen Sozialisation und fachkulturellen Bedingungen stets in einer Pluralität auf, was zu einer disunity of science und Inkommensurabilitäten zwischen den Träger/innen der wissenschaftlichen Stile führt (Hacking, 1985). 
randin in einem ero-epischen Gespräch ${ }^{12}$ über ihr Forschungsprojekt im Bereich der Katalyseforschung ausdrückt.

\begin{abstract}
Prof. Wolf-Ferrari: Manche Wissenschaften sind wie das SCHACHspiel. Da gibt's kein großes Problem, das Problem ist ja, dass man den König schlägt. Das ist kein großes Problem aber auf dieses Alltagsproblem gibt es im Schachspiel große ANTWORTEN! Und auch in der CHEMIE-also der organischen Chemie oder so-gibt es keine großen Probleme. Aber es gibt große Antworten. Dann gibt es aber Probleme in anderen Disziplinen, in denen gibt es eben auch GROSSE Fragen. (1) Früher hat man in der Physik zum Beispiel gesagt „was sind die elementarsten Einheiten, die es gibt?"
\end{abstract}

Die Nähe zur Physik wird darüber hinaus strategisch in der inner-chemischen Grenzarbeit genutzt, um ein hierarchisches Verhältnis zwischen härteren und weicheren chemischen Fachbereichen zu konstitutieren. Die implizite, normative Vorstellung einer Hierarchie der Naturwissenschaften, die sich bezüglich ihrer Objektivität, Härte und Reinheit der Erkenntnis anordnen lassen (Stengers, 2010c), ist in den laufenden Bemühungen, sich im im Gefüge der Naturwissenschaften $\mathrm{zu}$ positionieren, omnipräsent. Dies zeigt sich etwa in Laborsituationen, in denen Vertreter/innen der anorganischen Chemie hervorheben, dass sie dieselben analytischen Geräte und Messmethoden verwenden „wie die Physiker“, die zudem in der Anschaffung deutlich kostspieliger sind als die Laborausstattung der organischen Chemie. Wie in Abschnitt 2.3 deutlich wurde, gilt die Mittelakquise für kostenintensive Geräte als wichtige Kapitalie im Feld der Chemie und als Karrieretreiber. Darüber hinaus werden die in ihrer Materialität reduzierten, unscheinbaren, sauberen und geruchlosen Methoden der anorganischen bzw. physikalischen Chemie denjenigen der organischen Chemie gegenüber als überlegen inszeniert. Die Methoden der wet chemistry gelten als komplex, zufällig, wenig kontrollierbar und viel zu sehr vom Geschick des/der ausführenden Chemikers/in anhängig. Im Gegensatz dazu erzeugen Geräte wie das Rastertunnelmikroskop oder das Röntgendiffraktometer eindeutigere Ergebnisse, die sich zudem direkt aus den zugrundeliegenden quantenphysikalischen Dynamiken und Gesetzmäßigkeiten der Materie ergeben.

Die Grenzgänge von Chemiker/innen, die sich in den Material- und Nanowissenschaften betätigen und über entsprechende Anbindungen an physikalische wie Lebenswissenschaften verfügen, finden nachhaltigen Niederschlag in den basalen 
Konzeptionen der molekularen Welt - und namentlich der Chiralität. So fällt auf, dass entsprechende Chemiker/innen in der Interviewstudie einen deutlich weiter gefassten Chiralitätsbegriff vertreten als vergleichbare Kolleg/innen in anderen chemischen und außer-chemischen Bereichen. Die epistemischen wie ontischen Bezüge gehen weit über die Konzeption der organischen Molekülstruktur hinaus und werden in einen Schilderungsduktus eingereiht, der die Universalität der Rechts-Links-Asymmetrie in der Natur betont (vgl. Abschnitt 6.2). Physikalische, kristallografische, anorganisch-chemische und auch biochemische Bezüge werden gleichwertig behandelt und stehen für die Einheit der Natur, die sich in makroskopischen Objekten wie auch auf allen anderen Ebenen als asymmetrisch erweist und chirale Phänomene hervorbringt. Das Weltbild von einer objektiv gegebenen Realität, die sich in emergente Systeme und Betrachtungsebenen aufschichtet und die Präidee der Einheitswissenschaft sind auch unter Chemiker/innen in den Feldern der Nano-, Material- und Oberflächenforschung omnipräsent. Es wird dabei deutlich, wie sehr Emergenz- und Reduktionstheorien auch den chemischen Erkenntnisprozess leiten, denn sie bieten in einem heterogenen, ausdifferenzierten und hybriden Bereich ein gewisses Maß an Orientierung: So wird in den Schilderungen der Befragten stets danach unterschieden, in welchem Bereich bzw. welcher Größenordnung und Auflösung der Natur man sich gerade bewegt und auch die eigene, alltägliche Auseinandersetzung mit der Chiralität wird innerhalb dieser eingeordnet.

Aus der Nähe zur Physik ergeben sich für die chemischen Bereiche der Materialwissenschaften nicht nur Erfolge im Einwerben von Ressourcen und eine prestigeträchtige Position im hierarchisch imaginierten Gefüge der Naturwissenschaften. Einige Chemiker/innen nehmen im Alltag eine zunehmende Aneignung und Umdeutung etablierter chemischer Gegenstände und Konzeptionen durch die physikalischen Wissenschaften wahr. Die Überschneidungen mit den physikalischen Feldern werden dabei durchaus ambivalent verhandelt: Auf der einen Seite bieten diese erhöhte Chancen auf Teilhabe an ressourcenintensiven Big Science-Projekten, andererseits erkennen Chemiker/innen auch, dass sie längst nicht mehr über ein Monopol in der Erforschung der molekularen Welt verfügen. Mit dem Aufkommen verschiedener mikroskopischer Betrachtungsebenen der Natur im Zuge der Physikalierung der MSE verlor die Heuristik der dreidimensionalen molekularen Struktur gegenüber zweidimensionalen Oberflächenmodellen sowie nano- und supramolekularen Zugriffen auf die Materie sukzessive an Bedeutung. Dies drückt sich u.a. in der Ausweitung der Bedeutung basaler molekularer Konzepte wie etwa der Chiralität aus, von der sich Chemiker/innen in einer Distinktion echter wie falscher Chiralität wie folgt abgrenzen:

Prof. Borodin: Die Chiralität hat sich jetzt in den Materialwissenschaften [durchgesetzt], bei den Physikern, bei Magnetleuten oder so, die benutzen das auch. Aber es 
gibt eine strikte Definition, wenn man so will. Von den Chemikern sag ich jetzt mal und die anderen Leute, die das benutzen, die scheren sich einen Dreck drum. Und benützen des einfach für allerlei Sachen, die SCHEINBAR spiegelsymmetrisch sind (2) oder die Spiegelsymmetrie brechen. Als Beispiel seh ich immer Magnetismus: Das sind ja bewegte Teilchen ein Elektron. Es gibt aber 'ne Definition die sagt wenn ich das gleiche erreichen kann durch Zeitumkehr dann ist es also das Spiegelbild dann ist es KEINE Chiralität und das ist genau beim Magnetismus der Fall wenn ich jetzt die die Bewegungsrichtung UMDREHE, also 'n Elektron hat ja 'n Spin das macht ja so [gestikuliert eine Rotationsbewegung]. Und wenn ich sage „jetzt dreh ich das rückwärts um und dann erreiche ich das gleiche Spiegelbild" dann ist das NICHT Chiralität! Das ist 'ne Definitionsfrage das kann jeder halten wie er will aber (1) aber wenn man die Definition ERNST nimmt, (1) dürfte man magnetische Systeme so nicht bezeichnen aber heutzutage schert sich keiner mehr um Regeln.

In der Rückaneignung und Verteidigung der Chiralität als genuin chemische Eigenschaft bedienen sich Chemiker/innen der Strategie der Historisierung. Mit dem Verweis auf die Geschichte der Chiralität und die zentralen Meilensteine ihrer Entdeckung wird dabei die Bedeutung der (organischen) Chemie hervorgehoben, allen voran im Namen der Pioniere des Feldes wie Louis Pasteur, Joseph Achille Le Bel und Jacobus Henricus Van 't Hoff, die sich insbesondere um die Stereochemie verdient gemacht hatten. Frühe optische wie kristallografische Beiträge zur theoretischen wie empirischen Begründung der Chiralitätsforschung, die eher der Physik zuzurechnen sind, werden dabei allerdings ebenfalls gewürdigt. Die Geschichte der Chiralität wäre zudem auch nicht ohne die Erkenntnisse der Teilchenphysik zu den fundamentalen Asymmetrieverhältnissen der Materie denkbar:

Prof. Borodin: [Der Begriffder Chiralität] wurde in den Naturwissenschaft eingeführt durch Lord Kelvin [...] [um] 1890, ein gebildeter Physiker, DER das eingeführt hat eben für die Händigkeit in Materialien oder in Kristallen oder in Molekülen. [...] Der Begriff wurde aber nicht benutzt! Die Chemiker hatten damals weiterhin von Pasteur's Dissymmetrie gesprochen. Irgendwann in den sechziger Jahren hatte man in der Kernphysik die Paritätsverletzung entdeckt und DORT (.) wurde wiederholt von jemandem vorgeschlagen, man sollte das auch richtig BENENNEN. Man hat dann Werbung für das Wort Chiralität gemacht, das DANN in den sechziger Jahren wenige berühmte Chemiker aufgegriffen haben und dann hat sich das so langsam durchgesetzt in der Chemie. (3) Dann war's eigentlich nur in der Kernphysik und in der Chemie gebraucht worden (2) und hat sich jetzt in den Materialwissenschaften [durchgesetzt], bei den Physikern, bei Magnetleuten oder so, die benutzen das auch. Aber es gibt eine strikte Definition, wenn man so will. Von den Chemikern sag ich jetzt mal und die anderen Leute, die das benutzen, die scheren sich einen Dreck drum. 
Wie diese historisierende Schilderung deutlich macht, besteht im Bewusstsein über die Geschichte des eigenen Faches ein gefestigtes Narrativ zur Geschichte der Chiralität, in dem Physiker/innen sowie Vertreter/innen der organischen Chemie und Kristallografie einen gleichberechtigten Platz einnehmen. (Quanten-)Physikalische wie organisch-chemische Zugänge zur Chiralität werden dabei in der historisierenden Reflexion in einem harmonischen und produktiven Verhältnis geschildert, das allerdings in den 1980er Jahren erodierte, als eine experimentalphysikalische und ingenieurswissenschaftliche Aneigung des Chiralitätskonzepts stattfand und der Bedeutungshorizont der Chiralität eine Entgrenzung erfuhr. In der Aushandlung und Distinktion zwischen Chiralitätsdefinitionen „im strengen Sinne“ bzw. „in ihrer ursprünglichen Bedeutung“, wird versucht, die Chiralität als chemische Eigenschaft (wenn auch mit Anbindungen an physikalische Phänomene) zurückzuerobern. An dieser Stelle wird deutlich, dass diese Auseinandersetzungen mit der Rückaneignung bzw. Verteidigung basaler chemischer Konzeptionen und Begrifflichkeiten nicht nur um ihrer selbst Willen geführt werden. Vielmehr sind mit dem Besetzen von Begriffen wissenschaftliche Ressourcenfragen verbunden, was insbesondere dadurch deutlich wird, dass Chemiker/innen in diesem Zusammenhang die wirtschaftliche Bedeutung ihrer Entdeckungen und Erfindungen für das Wohl der Gesellschaft betonen. Auch dies erfolgt in einem historisierenden Duktus, wie etwa in folgender Beispielerzählung zur Bedeutung der Katalyseforschung:

Prof. Borodin: [Ich halte derzeit eine] Oberflächenvorlesung [zur Chiralität], bei der kommt die [chirale] Anordnung der Moleküle vor, wie sich Kristalle bilden, wie an ihren Oberflächen Prozesse ablaufen, Biomoleküle oder KLEINE Moleküle - wichtig ist Katalyse! Die Abgasreinigung vom Automobil zum Beispiel ist eine chemische Reaktion, die an einer Oberfläche-Platin, Eisen oder sowas-abläuft, die man aber nicht VERSTEHT! Das heißt, Forschung ist nach wie vor dringend notwendig, obwohl über zwanzig Prozent unseres Bruttosozialproduktes aufder Welt durch ReakTIONEN an Oberflächen zustandekommt, wissen wir wenig darüber. Die Menschheit wäre heute nicht [da], wo sie ist wenn nicht Haber und Bosch die Ammoniaksynthese erfunden hätten - die Aufspaltung von Stickstoff in DÜNGER wenn man so will. ]a, [...] das hat nen RIESICEN Fortschritt der Menschheit gebracht. Das wird heute immer unterSCHÄTZT.

Der zitierte Vertreter der Katalyseforschung und Oberflächenchemie betont den kreativen, schöpferischen Aspekt chemischen Schaffens, dessen Entdeckungen in nützliche Anwendungen münden. Er setzt die Bemühungen der gegenwärtigen Katalyseforschung in eine genealogische Linie mit Fritz Haber und Carl Bosch, die mit der Ammoniaksynthese einen besonders gewichtigen Beitrag im Namen dieses Kollektivs leisteten. Die industriell wie wissenschaftlich tätigen Chemieingenieur/innen werden in diesem Duktus in den Dienst ganzer Nationen gestellt, 
indem deren Bedeutung für Wirtschaftswachstum, Wohlstand und Fortschritt betont werden. Allerdings bestehe eine erhebliche Diskrepanz zwischen ökonomischer Bedeutung der Chemie und ihrer gesellschaftlichen Wahrnehmung und Würdigung: Auch wenn Physiker/innen und Optiker/innen als Hilfe für die Chemiker/innen bei der Entdeckung der Chiralität und den Erkenntnissen und Anwendungen eine nicht unerhebliche Rolle spielen, werden im öffentlichen Interesse an den Naturwissenschaften die Beiträge der Physik ungleich stärker rezipiert und gewürdigt als diejenigen der Chemie. Sowie das Haber-Bosch-Verfahren als auch die Entdeckung der Chiralität werden dabei gleichermaßen in einem Narrativ der Verkanntheit verhandelt, das sich an der mangelnden Kenntnisnahme und Würdigung chemischer Erkenntnisse durch die Gesellschaft ausdrückt.

Darüber hinaus zeigt die Interviewstudie, dass die Stärkung des öffentlichen Bewusstseins für die Chemie insbesondere solchen Vertreter/innen der Chemie am Herzen liegt, die in hybriden Grenzbereichen mit der Physik beschäftigt sind. Das zeigt sich etwa in einem verstärkten interessement, sprich bei der Generierung von wissenschaftlichen Ressourcen wie Forschungsgelder, Räumlichkeiten und Nachwuchs strategisch auf die Aufmerksamkeit einer interessierten Öffentlichkeit $\mathrm{zu}$ fokussieren (Callon, 1999, S. 92). Die interviewten Vertreter/innen der entsprechenden Felder zeigen sich im Alltag darum bemüht, die Inhalte der Chemie zu popularisieren. So engagieren sich Interview-Partner/innen wie Prof. Borodin und Prof. Pejačević regelmäßig an Veranstaltungen von Wissenschaftsmuseen, Tagen der offenen Tür, halten populärwissenschaftliche Vorträge oder nutzen Onlineformate zur Popularisierung chemischer Themen und Belange. Die Chiralität ist dabei ein beliebter Anker in der Chemiekommunikation, insbesondere dadurch, dass sie sich als Universalismus der Natur rahmen lässt und Anknüpfungspunkt an andere wissenschaftliche Disziplinen und Alltagsbeispiele bietet. Die Chiralität wird dabei als genuin chemisches Konzept verhandelt, das die Bedeutung chemischen Wissens in der Gesellschaft hervorheben soll.

Im hybriden Bereich der Nano-, Material- und Oberflächenforschung fällt auf, wie stark chemisches und physikalisches Wissen, Handeln und styles of reasoning neben ingenieurswissenschaftlichen Wissensformen miteinander verwoben sind und kaum von einander getrennt betrachtet werden können. In diesem hybriden Bereich lassen sich die Grenzziehungsdynamiken von physikalischen wie chemischen Konzeptionen der molekularen Welt untersuchen. Dabei werden physikalische Konzeptionen zunehmend dominant und verdrängen die chemischen. Vor dem Hintergrund der Innovationsdispositive des NBIC-Paradigmas erfahren etablierte chemische Grundbegriffe wie die Chiralität in anwendungsorientierten Kontexten eine Umdeutung bzw. Begriffsverschiebung. Dabei zeigt sich, wie chemische Eigenschaften der mikroskopischen Struktur gegenüber als physikalisch begriffenen Aspekten wie Eigenschaften und Funktionen von Materialien verdrängt werden. Darüber hinaus zeigt sich, dass Vertreter/innen der Physik 
sich den Begriff der Chiralität aneignen und auf Phänomene beziehen, die sich der Definition im „strengen Sinne" entziehen. Auf diesem Wege verliert die Chemie epistemische Macht über ein traditionelles Anwendungsgebiet, aus dem sich bislang ein großer Teil der Forschungsinvestitionen und Ressourcen der Disziplin speisten. Ähnliches lässt sich in Feldern wie pharmazeutischen und biomedizinischen Anwendungsgebieten beobachten, in denen biowissenschaftliche Konzepte des Molekularen die klassische organischen Synthesechemie verdrängen und als nicht mehr zeitgemäß erscheinen lassen. 